\title{
Age modifiers for triage scores of older patients would not be helpful for emergency care
}

To the editor: I read with great interest the recent article by Hendin et al. that described the characteristics and outcomes of older patients who presented to the emergency department (ED) and were assigned a low acuity triage score. ${ }^{1}$ While the descriptions of these patients and outcomes were interesting, the conclusion that there should be consideration of an age modifier for triage scores is not supported by the data presented. As the authors acknowledged in the discussion, it is not a goal of triage scores to determine which patients will ultimately need admission. Patients would not benefit from a change in triage score because of their age based on a possible need for admission, if the current triage scores are accurately reflecting the needs of the patients in the ED. There is no suggestion in this study that there are concerns about the appropriateness of the scores for identifying the urgency at which care is needed for older patients in the ED. Previous work also indicates that the Canadian Triage and Acuity Scale (CTAS) has high validity for older patients. ${ }^{2}$ Acknowledging the special care needs of older patients is important, ${ }^{3}$ but up-triaging because of age alone is unlikely to benefit patients' emergency care. This proposed arbitrary up-triaging of older adults would only diminish the utility of ED triage systems.

Christopher R. Foerster, MD, $\mathrm{MSc}^{*}$

* Seaforth Community Hospital, Seaforth, ON

\section{REFERENCES}

1. Hendin A, Eagles D, Myers V, Stiell IG. Characteristics and outcomes of older emergency department patients assigned a low acuity triage score. CFEM 2018;20(5):762-9.

2. Lee JY, Oh SH, Peck EH, et al. The validity of the Canadian Triage and Acuity Scale in predicting resource utilization and the need for immediate life-saving interventions in elderly emergency department patients. Scand 7 Trauma Resusc Emerg Med 2011; 19:68.

3. Hwang U, Shah MN, Han JH, Carpenter CR, Siu AL, Adams JG. Transforming emergency care for older adults. Health Aff (Millwood) 2013;32(12):2116-21. 